\title{
Differences in Anthropometry, Biological Age and Physical Fitness Between Young Elite Kayakers and Canoeists
}

\author{
by \\ Daniel López-Plazaํ, Fernando Alacid ${ }^{1}$, José María Muyor², \\ Pedro Ángel López-Miñarro ${ }^{3}$
}

The aim of this study was to determine the anthropometric and physical characteristics of youth elite paddlers and to identify the differences between kayakers and canoeists. A total of 171 male paddlers (eighty-nine kayakers and eighty-two canoeists), aged $13.69 \pm 0.57$ years (mean \pm SD) volunteered to participate in this study. The participants completed basic anthropometric assessments (body mass, stretch stature, sitting height, body mass index, maturity level, sum of 6 skinfolds and fat mass percentage) as well as a battery of physical fitness tests (overhead medicine ball throw, counter movement jump, sit-and-reach and $20 \mathrm{~m}$ multi-stage shuttle run tests). The anthropometric results revealed a significantly larger body size (stretch stature and sitting height) and body mass in the kayakers $(p<0.01)$ as well as a more mature biological status $(p=0.003)$. The physical fitness level exhibited by the kayakers was likewise significantly greater than that of the canoeists, both in the counter movement jump and estimated VO2max ( $p<0.05)$, as well as in the overhead medicine ball throw and sit-and-reach test $(p<0.01)$. These findings confirm the more robust and mature profile of youth kayakers that might be associated with the superior fitness level observed and the specific requirements of this sport discipline.

Key words: anthropometry, physical fitness, biological age, kayak, canoe.

\section{Introduction}

Systematic sport training has been related to the development of certain physical attributes along with specific changes in the morphological characteristics of athletes (Gabbett and Georgieff, 2007; Ross and Marfell-Jones, 1991). Although a complex group of different variables favours performance in a given sport, there are some attributes which seem to be common in the most successful athletes (Leone et al., 2002). Over the past few years, research into the relationship between anthropometry and performance has increased (Gabbett and Georgieff, 2007; MielgoAyuso et al., 2015). In most sports, the athletes' overall status may be determined by means of general and specific field tests, since a strong correlation has been consistently reported between the fitness level and the individual performance attained (Pyne et al., 2006; van Someren and Howatson, 2008). Traditionally, the determination of a physical profile in a given sport involves the use of predictive testing as a measure of power and strength (Cronin and Hansen, 2005), speed (Gabbett and Georgieff, 2007), aerobic fitness (Leone et al., 2002) or flexibility (Simoneau, 1998). Along with measurements of body dimensions, predictive

\footnotetext{
1 - Department of Physical Activity and Sports, Catholic University of San Antonio, Murcia, Spain.

2 - Faculty of Education Sciences, Nursing and Physiotherapy. Laboratory of Kinesiology, Biomechanics and Ergonomic (KIBIOMER), University of Almería, Almería, Spain.

3 - Department of Physical Education. University of Murcia, Murcia, Spain.
} 
fitness tests provide an appraisal of the structural and physical status that may be used to describe the 'typical' successful athlete in a given sport (Ross and Marfell-Jones, 1991).

Within particular sports, there exist various disciplines or playing positions with specific demands that require different approaches in training and are associated with different physical and morphological characteristics (Gabbett and Georgieff, 2007; Mielgo-Ayuso et al., 2015). Sprint canoeing is a cyclic sport which consists of two disciplines kayaking and canoeing - both aiming to cover a specific distance as quickly as possible, and crossing the finish line before the opponents (Aitken and Neal, 1992; Shephard, 1987). From a biomechanical perspective, movement in kayaking consists of double-blade paddle cyclic movements on both sides of the boat, coordinated through pedalling movements and trunk rotation in a seated position, whereas canoeing consists of single-blade paddle cyclic movements performed on the same side of the boat from a kneeling position (up on one knee). Although there have been relatively few studies comparing the anthropometric attributes of both disciplines, the majority have agreed on the greater size and body mass of the kayakers (Arlettaz et al., 2004; Hirata, 1977). Conversely, a trend towards a larger thigh girth has been exhibited in canoeists, which might be related to the greater sum of 8 skinfolds observed in these athletes (Alacid et al., 2015; Ridge et al., 2007).

Traditionally, research into kayaking is primarily focused on physiological testing of the athletes in order to determine fitness levels and then designing training programs to optimize physiological fitness (Aitken and Neal, 1992). Early studies only analysed VO2max to monitor and assess the physiological capacity of elite kayakers (Pendergast et al., 1979; Tesch et al., 1976). Nevertheless, the measurement of maximal oxygen uptake of paddlers is not the only possible determinant of performance. While characteristics of the sport demand that kayakers paddle most of the race at or around peak VO2 (Bishop et al., 2002), requiring high aerobic power, the anaerobic aspects should not be overlooked (Fry and Morton, 1991; Tesch et al., 1976). Other variables apart from VO2 have been associated with optimal performance in paddling (Pendergast et al., 1979; Tesch et al., 1976). Fry and Morton (1991) using a battery of anthropometric and physiological tests, determined the most important attributes of elite sprint kayakers. Anthropometric variables such as muscle mass, height, body fat, and limb length have been identified as factors contributing to obtain optimal performance (Fry and Morton, 1991; Shephard, 1987; Sklad et al., 1994). The relationship between anthropometry and performance has also been confirmed by other studies (Ackland et al., 2003; Gobbo et al., 2002; van Someren et al., 2001) in an attempt to determine elite kayaking profiles in seniors and juniors, while in canoeing this relationship has not yet been studied, making a comparison between disciplines impossible.

Research into sprint paddling has focused only on investigating each variable separately, and has never taken field-based testing into consideration in the determination of paddler profiles, offering only a limited picture of the overall status of the athletes. Therefore, the purpose of this study was to identify the anthropometric and physical profile of youth elite paddlers competing at a high level and to compare them between disciplines. It was hypothesized that kayakers and canoeists would have different anthropometric and physical characteristics, as a result of different demands of each sport discipline.

\section{Material and Methods}

\section{Participants}

A total of 171 youth male paddlers (eighty-nine kayakers and eighty-two canoeists), aged $13.69 \pm 0.57$ years (mean $\pm \mathrm{SD}$ ), with training experience of $3.80 \pm 1.78$ and $2.51 \pm 1.38$ years (mean $\pm \mathrm{SD}$ ), respectively, participated in this study. The inclusion criteria were (a) training on regular basis between 4 and $6 \mathrm{~d} \cdot \mathrm{wk}^{-1}$, (b) at least 2 hours of daily training and (c) being selected that year by the Royal Spanish Canoeing Federation as the best in their age category to participate in National Development Camps between 2005 and 2008. The Institutional Ethics Committee of the Catholic University of San Antonio approved the study and a signed written informed consent form was obtained from the participants and their parents before the beginning of testing. Any participant reporting illness or pharmacological treatment during the 
testing period was excluded from the study.

\section{Procedures}

A series of physical and anthropometric tests was performed over a 3 day period at the National Development Camps. Before the beginning of each physical test, clear instructions were given to all participants, as well as a warmup time consisting of 6-8 minutes of multidirectional running and 5 minutes of upper and lower limb general dynamic stretching supervised by a strength coach. A 5-minute familiarisation time with the materials and procedures was also provided as part of the specific warm-up for each test. The testing session began with anthropometric assessments followed by upper and lower body physical tests to prevent any potential body composition changes (Gabbett and Georgieff, 2007). In addition, the participants were required to abstain from intensive training sessions 48 hours before the National Camps and retain their normal pre-training diet prior to testing.

Anthropometry

Anthropometric variables included age (years), body mass $(\mathrm{kg})$, stretch stature $(\mathrm{cm})$, sitting height $(\mathrm{cm})$, and the sum of 6 skinfolds $(\mathrm{mm})$ (triceps, subscapular, supraspinale, abdominal, front thigh and medial calf), and were measured following the guidelines described by the International Society for the Advancement of Kinanthropometry (ISAK) (Stewart et al., 2011). Body mass was evaluated using a SECA 862 scale (SECA, Germany); stretch stature and sitting height were determined with a GPM anthropometer (Siber-Hegner, Switzerland), and skinfolds with a Harpenden skinfold calliper (British Indicators, UK). All instruments were calibrated at the beginning of each testing session to prevent measurement errors. A fully certified Level-2 ISAK anthropometrist measured each variable two or three times, if the difference between the first two measurements were greater than $5 \%$ for the skinfolds and $1 \%$ for the rest of the dimensions, with the mean values (or median in the last case) used for further data analysis. The intra-rater technical error of measurement was set at $3.05 \%$ for the skinfolds and $0.69 \%$ for the other variables. The body mass index (BMI) was determined by the equation: body mass $(\mathrm{kg}) /$ stretch stature $2(\mathrm{~m})$, while fat mass content (\%) was calculated following the procedures defined by Slaughter et al. (1988), which take into consideration the sum of 6 skinfolds. The measurements showed an intra-class correlation coefficient (ICC) of 0.85 for test-retest reliability and a coefficient of variation (CV) of $3.8 \%$.

Maturity

Biological maturity was estimated for each participant according to the procedures described by Mirwald et al. (2002). The age at peak height velocity (APHV) was considered as a maturational benchmark ( 0 value) and each measurement was described as years from peak height velocity (PHV), assuming the difference in years as a value of the maturity offset.

Upper body power assessment

To evaluate upper body muscular power, the Overhead Medicine Ball Throw test (OMBT) was performed using a 3-kg medicine ball (Gabbett and Georgieff, 2007; Mielgo-Ayuso et al., 2015). From a standing and arm-relaxed position the participants were instructed to throw the ball as far forward as possible. Counter movements were allowed as long as the feet were not moved during the test. The distance of the throw was recorded to the nearest centimetre, taking for analysis the best of 3 throws with at least $2 \mathrm{~min}$ rest between attempts. The measurements showed an intra-class correlation coefficient (ICC) of 0.95 for test-retest reliability and a coefficient of variation $(\mathrm{CV})$ of $3.2 \%$.

Lower body power assessment

The Counter Movement Jump test (CMJ) was used for the determination of lower body strength following the recommendations described by Temfemo et al. (2009). All jumps were performed on a Bosco platform (Bosco System) which recorded athletes' contact time ( $\mathrm{m}$. $\mathrm{s}^{-1}$ ). A counter movement until approximately $90^{\circ}$ of knee flexion was allowed prior to the jump. The best of 3 attempts, with at least 3 min rest inbetween was recorded for posterior Jump height (m) calculations. An intra-class correlation coefficient (ICC) of 0.99 for test-retest reliability and a coefficient of variation $(\mathrm{CV})$ of $2.2 \%$ were shown by the CMJ test.

Flexibility

A Sit-and-Reach test (SR) was selected to determine hamstring flexibility. The participants were required to sit with their legs together and knees extended with heels flat against the bottom of a testing board (Richflex System, Sportime, 
Atlanta). By sliding their hands together one over the other, participants were asked to slowly reach as far forward as possible along the testing board and to hold the resulting position for at least two seconds. The examiner then registered the distance reached to the nearest centimetre by means of a tape measure placed on the top of the board with the zero mark representing the plantar surface. Therefore, positive values were considered once participants had reached beyond their toes. The best result of 3 attempts was recorded for further analysis, with a rest time between attempts of at least 3 minutes. The measurements showed an intra-class correlation coefficient (ICC) of 0.90 for test-retest reliability and a coefficient of variation $(\mathrm{CV})$ of $2.9 \%$.

Maximum oxygen uptake

Maximal aerobic capacity $\left(\mathrm{VO}_{2 \max }\right)$ was estimated following the procedures described by Lager and Lambert (1982) for the multi-stage shuttle run test (mp3 version, Coachwise, UK). Each participant was required to perform a progressively faster 20-m shuttle run, being timed with an audible "beep", until reaching volitional exhaustion. If two consecutive shuttles were not completed in time, the participant was excluded for the next repetition; this being considered the end of the test. The last successful repetition made by the athlete was registered for subsequent $\mathrm{VO}_{2 \max }$ estimation using the regression equation defined by Ramsbottom et al. (1988). The measurements showed an intra-class correlation coefficient (ICC) of 0.92 for test-retest reliability and a coefficient of variation $(\mathrm{CV})$ of $2.6 \%$.

\section{Statistical analysis}

The hypotheses of normality and homogeneity of variance were verified using the Kolmogorov-Smirnov test and the Levene's test, respectively. When statistical tests revealed no violations of the assumptions of normality and homogeneity, the difference between the mean values between groups was analysed using a ttest for independent samples. The Mann-Whitney non-parametric test was used when normality supposition of data was rejected. The level of significance was set at $p<0.05$. Cohen's $d$ was used to measure the effect size of observed differences, and was considered small when between 0.2 and 0.5 , moderate when between 0.5 and 0.8 , and large when the effect was $>0.8$ (Cohen, 1988). All statistical analyses were conducted using SPSS v22.0 (SPSS Inc. Chicago IL, USA).

\section{Results}

The results of the anthropometric variables are summarised for each discipline (kayak and canoe) in Table 1. It can be observed that kayakers were significantly heavier and taller $(p<0.01)$ than the canoeists, showing small effect size in body mass (Cohen's $d=0.4$ ) and medium effect size values in stretch stature and sitting height (0.6 in both cases). The analysis also revealed a significantly greater maturity status in the kayakers $(p=0.003)$ when comparing the years from/to the age at peak height velocity $(0.48 \pm 0.76$ vs $0.10 \pm 0.91$ for kayakers and canoeists, respectively). Conversely, no differences between means or meaningful effect size values were found regarding the BMI, sum of skinfolds or fat mass percentage.

The results of the field based test variables in both kayakers and canoeists are presented in Table 2. Significantly greater values were observed in kayakers than in canoeists in the OMBT test $(6.09 \pm 1.31 \mathrm{~m}$ and $5.56 \pm 1.21 \mathrm{~m}$, respectively) and SR test $(8.49 \pm 6.17 \mathrm{~cm}$ and $3.47 \pm$ $7.77 \mathrm{~cm}$, respectively). Cohen's $d$ calculations revealed medium effect size values for both OMBT $(d=0.7)$ and SR $(d=0.4)$. Similarly, significantly higher values were detected in the CMJ and estimated $\mathrm{VO}_{2 \max }$ variables in the kayakers whereas the analysis of the effect size only revealed a medium effect value for $\mathrm{VO}_{2 \max }$ (Cohen's $d=0.5$ ).

\section{Discussion}

The main objective of this research was to determine the anthropometric and physical characteristics of youth elite paddlers. It should be highlighted that this is the first comparative interdisciplinary study between kayaking and canoeing. The main finding was the significantly greater physical fitness level and a more robust and mature anthropometric profile exhibited by the kayakers. These results provide normative data about the status of youth male paddlers competing at a high level which allow for the identification of an optimal profile for each discipline.

The basic anthropometric variables have been seen to be important when identifying the 
most talented paddlers (Ackland et al., 2003; Alacid et al., 2011; Ridge et al., 2007). Considering the anthropometric results of the present study, kayakers revealed a significantly taller and heavier profile than canoeists. These differences in the stretch stature $(4-5 \mathrm{~cm})$ and body mass $(4-5 \mathrm{~kg})$ are in agreement with those reported in previous research (Hirata, 1977) that indicated even greater variations in youth male paddlers (approximately 8-9 $\mathrm{cm}$ and 6-9 $\mathrm{kg}$, respectively) (Arlettaz et al., 2004). When kayakers' and canoeists' results are compared separately with studies of other age groups, analogous values are obtained in the stretch stature and body mass (Alacid et al., 2011; Cuesta et al., 1991) as well as in sitting height (Alacid et al., 2011, 2015). Previous studies conducted on Olympic and other elite paddlers reported BMI values no lower than $23 \mathrm{~kg} \cdot \mathrm{m}^{-2}$ (Ackland et al., 2003; Gobbo et al., 2002; Hirata, 1977), which are far beyond those observed in the current investigation $\left(20.9\right.$ and $20.6 \mathrm{~kg} \cdot \mathrm{m}^{-2}$ for kayakers and canoeists, respectively), perhaps due to the larger lean mass and robust somatotypes revealed in elite adult paddlers (Ackland et al., 2003; Alacid et al., 2011; Ridge et al., 2007). Furthermore, the BMI and lean body mass, along with other basic anthropometric variables such as the stretch stature and body mass have been positively related to better performance not only in kayaking and canoeing (Fry and Morton, 1991; van Someren and Palmer, 2003), but also in rowing (Sklad et al., 1994). However, no performance data were collected in the current study to corroborate this relationship. Comparing the current research results with previous studies conducted on youth paddlers, similar patterns can be observed, as canoeists presented slightly lower BMI values than kayakers, reaching values below $22 \mathrm{~kg} \cdot \mathrm{m}^{-2}$ (Alacid et al., 2011; Cuesta et al., 1991). Nonetheless, the importance of compact and robust somatotypes for the most successful sprint paddlers has been strongly supported, as mentioned above, and should be taken into consideration as a factor for talent identification.

\begin{tabular}{|c|c|c|c|c|c|c|}
\hline \multicolumn{7}{|c|}{$\begin{array}{l}\text { Table } 1 \\
\text { Mean values }( \pm \text { SD) and 95\% confidence intervals for the means of the } \\
\text { anthropometric variables and maturity status in kayakers and canoeists }\end{array}$} \\
\hline & \multicolumn{2}{|l|}{ Kayak } & \multicolumn{2}{|l|}{ Canoe } & \multirow{2}{*}{$p$} & \multirow{2}{*}{$\begin{array}{l}\text { Effect } \\
\text { size } \\
\text { (Cohen's d) }\end{array}$} \\
\hline & Mean \pm SD & $95 \%$ CI & Mean \pm SD & $95 \%$ CI & & \\
\hline Age (years) & $13.68 \pm 0.55$ & $13.56-13.80$ & $13.69 \pm 0.60$ & $13.56-13.80$ & 0.767 & 0.1 \\
\hline Body mass (kg) & $59.79 \pm 9.50$ & $57.73-61.85$ & $55.45 \pm 12.17$ & $52.72-58.17$ & 0.008 & 0.4 \\
\hline Stretch Stature $(\mathrm{cm})$ & $168.59 \pm 6.80$ & 167.12 - 170.07 & $163.01 \pm 9.76$ & $160.82-165.19$ & $<0.001$ & 0.6 \\
\hline Sitting Height (cm) & $89.06 \pm 4.27$ & $88.14-89.99$ & $86.09 \pm 5.45$ & $84.87-87.31$ & $<0.001$ & 0.6 \\
\hline BMI (kg $\cdot \mathrm{m}-2)$ & $20.94 \pm 2.37$ & $20.43-21.46$ & $20.64 \pm 2.93$ & $19.98-21.29$ & 0.125 & 0.1 \\
\hline Sum of 6 skinfolds & $64.31 \pm 24.80$ & $58.93-69.70$ & $64.77 \pm 34.43$ & $57.06-72.48$ & 0.150 & 0.1 \\
\hline $\begin{array}{l}\text { Fat mass percentage } \\
(\%)\end{array}$ & $15.88 \pm 5.66$ & $14.72-17.03$ & $15.58 \pm 7.67$ & $14.06-17.30$ & 0.150 & 0.1 \\
\hline $\begin{array}{l}\text { Maturity (years } \\
\text { from/to APHV) }\end{array}$ & $0.48 \pm 0.76$ & $0.32-0.65$ & $0.10 \pm 0.91$ & $-0.11-0.30$ & 0.003 & 0.5 \\
\hline
\end{tabular}

APHV: Age at Peak Height Velocity; BMI: Body Mass Index 


\begin{tabular}{|c|c|c|c|c|c|c|}
\hline \multicolumn{7}{|c|}{$\begin{array}{c}\text { Table } 2 \\
\text { Mean values }( \pm S D) \text { and 95\% confidence intervals for the means of the physical fitness } \\
\text { variables in kayakers and canoeists }\end{array}$} \\
\hline & \multicolumn{2}{|l|}{ Kayak } & \multicolumn{2}{|l|}{ Canoe } & \multirow{2}{*}{$\mathrm{p}$} & \multirow{2}{*}{$\begin{array}{l}\text { Effect size } \\
\text { (Cohen's d) }\end{array}$} \\
\hline & Mean \pm SD & $95 \% \mathrm{CI}$ & Mean \pm SD & $95 \% \mathrm{CI}$ & & \\
\hline OMBT (m) & $6.09 \pm 1.31$ & $5.81-3.38$ & $5.56 \pm 1.21$ & $5.29-5.83$ & 0.009 & 0.4 \\
\hline $\mathrm{CMJ}(\mathrm{m})$ & $0.36 \pm 0.07$ & $0.34-0.37$ & $0.34 \pm 0.07$ & $0.32-0.35$ & 0.035 & 0.3 \\
\hline $\mathrm{SR}(\mathrm{cm})$ & $8.49 \pm 6.17$ & $7.15-9.83$ & $3.47 \pm 7.77$ & $1.73-5.21$ & $<0.001$ & 0.7 \\
\hline $\begin{array}{l}\mathrm{VO} 2 \mathrm{max} \\
(\mathrm{ml} \cdot \mathrm{kg}-1 \cdot \min -1)\end{array}$ & $50.43 \pm 4.73$ & $49.41-51.46$ & $47.88 \pm 4.84$ & $46.80-48.97$ & 0.049 & 0.5 \\
\hline \multicolumn{7}{|c|}{$\begin{array}{c}\text { CMJ: Counter Movement Jump; OMBT: Overhead Medicine Ball Throw; SR: Sit and } \\
\text { Reach }\end{array}$} \\
\hline
\end{tabular}

The level of adiposity plays an important role in the total paddler-boat weight since it directly affects the boat submerged area and increases friction drag which may cause decreases in boat's speed (Alacid et al., 2011; Jackson, 1995). In the current study, young kayakers presented no significant differences in the percentage of fat mass and the sum of 6 skinfolds compared to canoeists. Unfortunately, not many comparisons between both disciplines have been conducted in the literature, focusing instead on gender differences and the paddling level (Fry and Morton, 1991; Sidney and Shephard, 1973). Previous studies of youth kayakers reported lower adiposity values to those described here, ranging from 6 to 13\% (Arlettaz et al., 2004; Cuesta et al., 1991; Gobbo et al., 2002; Sidney and Shephard, 1973). When observing elite adult paddlers, greater adiposity $(14.1 \%)$ and sum of 6 skinfolds were identified by van Someren and Palmer (2003) among the most successful paddlers. Conversely, Fry and Morton (1991) detected that the greater fat mass, the poorer the race time achieved in $1,000 \mathrm{~m}$ and $500 \mathrm{~m}$ events, and also found a negative relationship between body fat and performance as race distance increased. There is evidence to suggest that the age and the nature of the event are determinants in adiposity levels, since older and shorter event paddlers presented larger fat mass values (Fry and Morton, 1991; Sidney and Shephard, 1973; van Someren and Palmer, 2003). From the several equations for estimating the fat mass percentage, the formula described by Slaughter et al. (1988) was selected, since it was considered the most accurate in measuring the youth population (Mendez-Villanueva et al., 2011). However, any kind of comparison between studies must be treated with caution due to the different methods for estimating the percentage of fat mass. In other water sports such as swimming, the fat mass values of youth athletes seem to be lower (Laett et al., 2010). Perhaps this fact and the evidence of large body mass and BMI variations observed between elite paddlers (Ackland et al., 2003) might indicate that the morphological characteristics of the athletes are not as much of a determinant of performance as in other sports, where the body has to perform movements in direct contact with the particular physical environment of the sport discipline.

An analysis of maturation is especially 
important in individual sports where the physical level is paramount in the attainment of optimal performance (Vaeyens et al., 2008). Relatively few studies into maturation suggest a development of superior physical attributes in the most biologically mature athletes at the same chronological age (Mendez-Villanueva et al., 2011; Mirwald et al., 2002; Vaeyens et al., 2008). Following Alacid et al. (2015), the biological maturation observed in the current research in kayakers was significantly higher than in canoeists, even more than it could be expected from the height and body mass variables. According to previous research conducted by Alacid et al. (2015), the superior biological maturation observed in kayakers compared to canoeists might be expected from the differences in body height and mass variables identified between disciplines.

Traditionally, all beginners start by learning the fundamentals of paddling in a kayak, and only later decide to either move to canoeing or remain and excel in the kayak. The decision to move to canoeing in youth paddlers is apparently influenced by maturity, since to achieve optimal performance in kayaking demands an early strong physical development, while canoeing involves more technical ability (Alacid et al., 2015). Therefore, it seems reasonable that athlete selection programs should take into account not only the performance level, but its relationship with maturation in order to ensure a complete picture of the paddlers' potential, and so as not to make premature decisions on athlete selection at young ages (Mirwald et al., 2002; Vaeyens et al., 2008; Welsman and Armstrong, 2000).

The importance of the fitness level has been demonstrated not only when describing the athletes' physical fitness profile, but also when identifying potential successful athletes for certain sports (Gabbett and Georgieff, 2007; Leone et al., 2002). This is the first study which analyses the fitness level of youth elite paddlers using a battery of field based tests, and which demonstrates the significantly superior level of physical fitness in the kayakers within all the tested variables. The OMBT and CMJ tests were used in accordance with previous studies as the better predictor of limb power (Gabbett and Georgieff, 2007; Temfemo et al., 2009). In fact, there was some evidence to suggest a meaningful correlation between the power production of the lower and upper limbs when performing explosive movements, as this depends on neural coordination and postural control (Debanne and Laffaye, 2011; Mayhew et al., 2005). Additionally, other factors associated with anthropometry and maturation may explain the better performance exhibited by the kayakers regarding the arm span, leg length and lean mass (Cronin and Hansen, 2005; Temfemo et al., 2009).

Hamstring flexibility is an important factor in the fitness level and the prevention of spinal injuries, and especially in kayaking where systematic trunk rotation along with lumbar flexion occur (López-Miñarro et al., 2008). The hamstring extensibility values obtained in the present study are similar to the findings observed in previous studies conducted on young paddlers, with slightly lower SR values not exceeding $6 \mathrm{~cm}$ for kayakers and $3 \mathrm{~cm}$ for canoeists (LopezMiñarro et al., 2008, 2013). The expected greater flexibility revealed in the kayakers might be determined by the great lumbar flexion used during the paddling action (López-Miñarro and Alacid, 2010), which is very different than the one used in canoeing. The SR test is an appropriate mean of determining spine flexibility and range of motion in the pelvic tilt, whereas its validity as a measure of hamstring flexibility has been reported as moderate (Muyor et al., 2014). While hamstring extensibility in kayakers exhibits no significant differences between legs, the kneeling position necessary in canoeing appears to be responsible for the greater values observed in the forward leg as opposed to the kneeling leg (Lopez-Miñarro et al., 2008, 2013). For these reasons the straight leg raise or knee extension tests are more appropriate. However, the sit-andreach test was used as a measure of hamstring flexibility because it represents an agile field test for large group assessments, and can be easily used by coaches (Simoneau, 1998). Thus, it seems desirable that stretching is included in training programmes (López-Miñarro and Alacid, 2010).

Maximum oxygen uptake has been the main physiological variable studied in the kayak literature due to its relationship with race times (Pendergast et al., 1979; Shephard, 1987; Tesch et al., 1976). However, in youth athletes it seems that VO2max values and performance in a given sport are not significantly related (Bar-Or, 1987). 
Unsurprisingly, the kayakers exhibited significantly larger estimated VO2max values that confirm their greater aerobic capacity. Expressing VO2max relative to body mass has also revealed superior aerobic endurance of the kayakers regardless of their size and higher maturity levels. Previous research had indicated significantly higher VO2max levels than those observed here in both ergometer and treadmill tests, reporting values not lower than $54 \mathrm{ml} \bullet \mathrm{kg}-1 \cdot \min -1$ in either case (Fry and Morton, 1991; Shephard, 1987; Sidney and Shephard, 1973). However, any kind of comparison between studies must be carefully regarded due to the different protocols applied to estimate oxygen uptake.

\section{Conclusions}

The current investigation demonstrated the kayaker's superior size and body mass that indicates more robust and compact morphology when compared to canoeists. Similarly, analysis of the fitness tests revealed a significantly greater fitness level in the youth kayakers compared to youth canoeists, which is perhaps a consequence of the lower maturity status of the latter. These findings confirm the hypothesis that the differences between kayakers and canoeists may be related to the different requirements of each sport discipline and biological status. Nevertheless, further research should be carried out in order to confirm these findings and investigate their relationship with on-water performance.

\section{Acknowledgements}

The authors would like to acknowledge the collaboration of all coaches and athletes who volunteered to participate in this research paper, and their willingness to help in the course of the testing sessions. We would also like to thank the Royal Spanish Canoeing Federation for its cooperation and support during the process.

\section{References}

Ackland TR, Ong KB, Kerr DA, Ridge B. Morphological characteristics of Olympic sprint canoe and kayak paddlers. J Sci Med Sport, 2003; 6: 285-294

Aitken DA, Neal RJ. An on-water analysis system for quantifying stroke force characteristics during kayak events. Int J Sport Med, 1992; 8: 165-173

Alacid F, Marfell-Jones M, Lopez-Minarro PA, Martinez I, Muyor JM. Morphological Characteristics of Young Elite Paddlers. J Hum Kinet, 2011; 27: 97-112

Alacid F, Marfell-Jones M, Muyor JM, López-Miñarro PA, Martínez I. Kinanthropometric Comparison between Young Elite Kayakers and Canoeists. Coll Antropol, 2015; 39: 119-24

Arlettaz A, Rieth N, Courteix D. Assessment of lean body mass and bone mass in specific sites in elite kayak paddlers. Sci Sport, 2004; 19: 199-201

Bar-Or O. A commentary to fitness and children: a public health perspective. Res Q Exerc Sport, 1987; 58: 304307

Bishop D, Bonetti D, Dawson B. The influence of pacing strategy on VO2 and supramaximal kayak performance. Med Sci Sports Exerc, 2002: 34: 1041-1047

Cohen J. Statistical power analysis for the behavioral science. 2nd ed. Hillsdale, NJ: Lawrence Erlbaum Associates; 1988

Cronin JB, Hansen KT. Strength and power predictors of sports speed. J Strength Cond Res, 2005; 19: 349-357

Cuesta G, Polo JM, Padilla S. Correlation between the performance time obtained in situ and the physiological parameters obtained on ergometer in young paddlers. Apunts, 1991; 18: 130-142

Debanne T, Laffaye G. Predicting the throwing velocity of the ball in handball with anthropometric variables and isotonic tests. J Sport Sci, 2011; 29: 705-713 
Fry RW, Morton AR. Physiological and kinanthropometric attributes of elite flatwater kayakists. Med Sci Sports Exerc, 1991; 23: 1297-1301

Gabbett T, Georgieff B. Physiological and anthropometric characteristics of Australian junior national, state, and novice volleyball players. J Strength Cond Res, 2007; 21: 902-908

Gobbo LA, Papst RR, Carvalho FO, Souza CF, Cuattrin SA, Cyrino ES. Anthropometric profile of the brasilian canoeing national team. Rev Bras Ci e Mov, 2002; 10: 7-12

Hirata K. Selections of Olympic champions. Tokio: Hirata Institute; 1977

Jackson PS. Performance prediction for Olympic kayaks. J Sport Sci, 1995; 13: 239-245

Laett E, Juerimaee J, Maeestu J, Purge P, Raemson R, Haljaste K, Keskinen KL, Rodriguez FA, Juerimaee T. Physiological, biomechanical and anthropometrical predictors of sprint swimming performance in adolescent swimmers. J Sport Sci Med, 2010; 9: 398-404

Lager L, Lambert J. A maximal multistage 20-m shuttle run test to predict. Eur J Appl Physiol O, 1982; 49: 1-12

Leone M, Lariviere G, Comtois AS. Discriminant analysis of anthropometric and biomotor variables among elite adolescent female athletes in four sports. J Sports Sci, 2002; 20: 443-449

Lopez-Miñarro PA, Muyor JA, Alacid F, Vaquero-Cristobal R, Lopez-Plaza D, Isorna M. Comparison of Hamstring Extensibility and Spinal Posture between Kayakers and Canoeists. Kinesiology, 2013; 45: $163-170$

López-Miñarro PA, Alacid F. Influence of hamstring muscle extensibility on spinal curvatures in young athletes. Sci Sport, 2010; 25: 188-193

López-Miñarro PA, Alacid F, Ferragut C, García A. Assessment and comparison of hamstring extensibility between young kayakers and canoeists. Motricidad. Eur J Hum Mov, 2008; 97-111

Mayhew JL, Bird M, Cole ML, Koch AJ, Jacques JA, Ware JS, Buford BN, Fletcher KM. Comparison of the backward overhead medicine ball throw to power production in college football players. J Strength Cond Res, 2005; 19: 514-518

Mendez-Villanueva A, Buchheit M, Kuitunen S, Douglas A, Peltola E, Bourdon P. Age-related differences in acceleration, maximum running speed, and repeated-sprint performance in young soccer players. $J$ Sport Sci, 2011; 29: 477-484

Mielgo-Ayuso J, Calleja-Gonzalez J, Clemente-Suarez VJ, Zourdos MC. Influence of anthropometric profile on physical performance in elite female volleyballers in relation to playing position. Nutricion Hospitalaria, 2015; 31: 849-857

Mirwald RL, Baxter-Jones ADG, Bailey DA, Beunen GP. An assessment of maturity from anthropometric measurements. Med Sci Sport Exer, 2002; 34: 689-694

Muyor JM, Vaquero-Cristobal R, Alacid F, Lopez-Minarro PA. Criterion-related validity of sit-and-rich and toe-touch tests as measure of hamstring extensibility in athletes. J Strength Cond Res, 2014; 28: 546-555

Pendergast D, Cerretelli P, Rennie DW. Aerobic and glycolytic metabolism in arm exercise. J Appl Physiol, 1979; 47: 754-760

Pyne DB, Duthie GM, Saunders PU, Petersen CA, Portus MR. Anthropometric and strength correlates of fast bowling speed in junior and senior cricketers. J Strength Cond Res, 2006; 20: 620-626

Ramsbottom R, Brewer J, Williams C. A progressive shuttle run test to estimate maximal oxygen uptake. $\mathrm{Br} \mathrm{J}$ Sport Med, 1988; 22: 141-144

Ridge B, Broad E, Kerr D, Ackland T. Morphological characteristics of Olympic slalom canoe and kayak paddlers. Eur J Sport Sci, 2007; 7: 107-113

Ross WD, Marfell-Jones M. Kinanthropometry. In: Physiological testing of the high performance athlete (2nd ed.), edited by MacDougal J, Wenger H and Green H. Champaign, IL: Human Kinetics, 223-308; 1991 
Shephard RJ. Science and medicine of canoeing and kayaking. Sports Med, 1987; 4: 19-33.

Sidney K, Shephard RJ. Physiological characteristics and performance of white-water paddler. Eur J Appl Physiol Occup Physiol, 1973; 32: 55-70

Simoneau GG. The impact of various anthropometric and flexibility measurements on the sit-and-reach test. J Strength Cond Res, 1998; 12: 232-237

Sklad M, Krawczyk B, Majle B. Body build profiles of male and female rowers and kayakers. Biol Sport, 1994; 11: $249-256$

Slaughter MH, Lohman TG, Boileau RA, Horswill CA, Stillman RJ, Van Loan MD, Bemben DA. Skinfold equations for estimation of body fatness in children and youth. Hum Biol, 1988; 60: 709-723

Stewart A, Marfell-Jones M, Olds T, de Ridder H. International standards for anthropometric assessment. New Zeland: ISAK: LowerHutt; 2011

Temfemo A, Hugues J, Chardon K, Mandengue S-H, Ahmaidi S. Relationship between vertical jumping performance and anthropometric characteristics during growth in boys and girls. Eur J Pediatr, 2009; 168: 457-464

Tesch P, Piehl K, Wilson G, Karlsson J. Physiological investigations of Swedish elite canoe competitors. Med Sci Sport, 1976; 8: 214-218

Vaeyens R, Lenoir M, Williams AM, Philippaerts RM. Talent identification and development programmes in sport - Current models and future directions. Sports Med, 2008; 38: 703-714

van Someren KA, Backx K, Palmer GS. The anthropometric and physiological profile of the international 200-m sprint kayaker. J Sports Sci, 2001; 19: 32

van Someren KA, Howatson G. Prediction of flatwater kayaking performance. Int J Sports Physiol Perform, 2008; 3: 207-218

van Someren KA, Palmer GS. Prediction of 200-m sprint kayaking performance. Can J Appl Physiol, 2003; 28: 505-517

Welsman JR, Armstrong N. Statistical techniques for interpreting body size-related exercise performance during growth. Pediatr Exer Sci, 2000; 12: 112-127

\section{Corresponding author:}

\section{Daniel López-Plaza, MSc}

UCAM, Universidad Católica San Antonio de Murcia

Campus de los Jerónimos s/n.

30107 Guadalupe, Murcia. Spain.

Phone: +34 968278824

E-mail: dlopez4@alu.ucam.edu 